\title{
Ultra-high energy cosmic rays in harmonic space
}

\author{
Stefano Camera ${ }^{a, b, c, *}$ \\ ${ }^{a}$ Dipartimento di Fisica, Università degli Studi di Torino, \\ Via P. Giuria 1, 10125 Torino, Italy \\ ${ }^{b}$ INFN - Istituto Nazionale di Fisica Nucleare, Sezione di Torino, \\ Via P. Giuria 1, 10125 Torino, Italy \\ ${ }^{c}$ INAF - Istituto Nazionale di Astrofisica, Osservatorio Astrofisico di Torino, \\ Strada Osservatorio 20, 10025 Pino Torinese, Italy \\ E-mail: stefano.camera@unito.it
}

I shall review our recent proposal of the use of the harmonic-space cross-correlation power spectrum between the arrival directions of ultra-high energy cosmic rays (UHECRs) and the distributions of galaxies in the Universe, as observed by cosmological surveys of the largescale structure (LSS). We expect the two observables to correlate, due to both galaxies and UHECR sources being hosted within dark matter haloes, which constitute the very LSS. This cross-correlation has not yet been considered in the literature. We formalise analytically such a cross-correlation and show how, if the distribution of UHECR sources traces indeed the LSS, the combination of auto- and cross-correlation greatly helps to detect UHECR anisotropies, even with current data. We show that the cross-correlation is more sensitive to UHECR anisotropies on small angular scales, more robust to systematic uncertainties, and it could be used to determine the redshift distribution of UHECR sources.

$37^{\text {th }}$ International Cosmic Ray Conference (ICRC 2021)

July 12th - 23rd, 2021

Online - Berlin, Germany

\footnotetext{
${ }^{*}$ Presenter
} 


\section{Introduction}

Ultra-high energy cosmic rays (UHECRs), defined as those cosmic rays (CRs) with energies exceding $1 \mathrm{EeV}\left(10^{18} \mathrm{eV}\right)$, have remained a mystery since their discovery [1], as we know not what they are, neither where they come from, nor the type of acceleration mechanism that is responsible for their formidable energies [e.g. 2, 3]. However, we do know that the highest energy rays are most likely extra-Galactic, for if they were produced within the Milky Way, their arrival directions in the sky would be very different from what we observe [4, 5]. Moreover, UHECR interactions with cosmological background photons produce the so-called Greisen-Zatsepin-Kuzmin limit-a sharp cutoff in the spectrum that is indeed observed in the data [6-8].

Then, if sources of UHECRs are extra-Galactic they should correlate with the cosmic largescale structure (LSS). Consequently, the UHECR flux distribution in the sky should present some degree of anisotropy, since the LSS is itself anisotropic [e.g. 9]. Here, I shall review a recent work where we introduce a new observable to the aim of detecting the anisotropy in the UHECR flux through the harmonic-space power spectrum of the cross-correlation between UHECR counts and the distribution of galaxies in the nearby Universe [10]. Such technique was previously proposed to study the anisotropy of the $\gamma$-ray sky [11-13], and proved successful for several tracers of the LSS [14-16]. If UHECR sources statistically trace the LSS, then the positions of these sources, and the arrival directions of UHECRs (if not strongly affected by intervening magnetic fields) should have a non-zero correlation with a galaxy sample up to a given distance.

Therefore, the detection or non-detection of the XC signal with galaxies at different redshifts would allow us to test whether UHECR sources are distributed according to the LSS, and to quantify to which extent the UHECR transfer function, determined by energy losses and intervening magnetic fields, does not depend on direction.

\section{Methodology}

Here, I introduce the main quantities and sketch the derivation of the equations entering the harmonic-space power spectrum (for both auto- and cross-probe correlations). For the full computation, we refer the reader to ref. [10]. Note that throughout this paper, CR energy losses will be assumed isotropic to first order.

\subsection{Observables}

\subsubsection{UHECRs}

Anisotropies in UHCR observed arrival directions $\hat{\boldsymbol{r}}$ above a certain energy cut $E_{\text {cut }}$ are

$$
\Delta_{\mathrm{CR}}\left(\hat{\boldsymbol{r}}, E_{\mathrm{cut}}\right):=\frac{\Phi\left(\hat{\boldsymbol{r}}, E_{\mathrm{cut}}\right)-\bar{\Phi}\left(E_{\mathrm{cut}}\right)}{\bar{\Phi}\left(E_{\mathrm{cut}}\right)},
$$

where $\bar{\Phi}\left(E_{\text {cut }}\right)$ is the sky-averaged UHECR flux. In turn, the number of UHECRs, detected in the direction $\hat{\boldsymbol{r}}$ above a given energy threshold $E_{\text {cut }}$ (in the observer's frame) and integrated over source redshifts reads

$\Phi\left(E_{\text {cut }}, \hat{\boldsymbol{r}}\right):=\int_{0}^{\infty} \mathrm{d} z \int_{E_{\text {cut }}}^{\infty} \mathrm{d} E_{\mathrm{o}} \frac{\mathrm{d} N}{\mathrm{~d} E_{\mathrm{o}} \mathrm{d} t \mathrm{~d} A \mathrm{~d} \Omega \mathrm{d} z}=\int \frac{\mathrm{d} z}{(1+z) H(z)} \frac{n_{\mathrm{s}, \mathrm{c}}(z, r \hat{\boldsymbol{r}})}{4 \pi} \int_{E_{\mathrm{cut}}}^{\infty} \mathrm{d} E_{\mathrm{o}} \frac{\mathrm{d} E_{\mathrm{e}}}{\mathrm{d} E_{\mathrm{o}}} E_{\mathrm{e}}^{-\gamma}$. 
Here: $\mathrm{d} N /\left(\mathrm{d} E_{\mathrm{o}} \mathrm{d} t \mathrm{~d} A \mathrm{~d} \Omega \mathrm{d} z\right)$ is the differential number counts of events per unit (observed) energy, unit time, unit detector area, unit solid angle on the sky, and unit redshift; $H(z)$ is the Hubble factor; $n_{\mathrm{s}, \mathrm{c}}$ is the volumetric number density of CR sources; and $r(z)$ is the radial comoving distance to redshift $z$, such that $\mathrm{d} r / \mathrm{d} z=1 / H(z)$. In Equation 2, the angle-averaged, isotropic emissivity of CRs is modelled as a power law, $E_{\mathrm{e}}^{-\gamma}$. Note that the emitted and observed energies are simply related by the redshifting of the photon frequency, i.e. $E_{\mathrm{o}}=E_{\mathrm{e}} /(1+z)$, if $z$ is the redshift at emission.

The number density of emitting sources can be written in terms of its average through the source-galaxy over-density field, namely $n_{\mathrm{s}, \mathrm{c}}(z, r \hat{\boldsymbol{r}})=\bar{n}_{\mathrm{s}, \mathrm{c}}(z)\left[1+\delta_{\mathrm{s}}(z, r \hat{\boldsymbol{r}})\right]$. Then, if we assume a non-evolving source population and introduce an attenuation factor $\alpha$, defined as the number of events reaching the Earth with $E_{\mathrm{o}}>E_{\text {cut }}$ divided by the number of events which would have reached the Earth if there were no energy losses, we have

$$
\Phi\left(E_{\mathrm{cut}}, \hat{\boldsymbol{r}}\right) \propto \frac{\bar{n}_{\mathrm{s}, \mathrm{c}} E_{\mathrm{cut}}^{1-\gamma}}{4 \pi(1-\gamma)} \int \mathrm{d} r \frac{\alpha\left(z, E_{\mathrm{cut}}\right)}{(1+z)}\left[1+\delta_{\mathrm{s}}(z, r \hat{\boldsymbol{r}})\right] .
$$

Hence, Equation 1 can be rewritten as

$$
\Delta_{\mathrm{CR}}\left(\hat{\boldsymbol{r}}, E_{\mathrm{cut}}\right)=\int \mathrm{d} r \phi_{\mathrm{CR}}(r) \delta_{\mathrm{s}}(z, r \hat{\boldsymbol{r}}),
$$

with $\delta_{\mathrm{s}}(z, r \hat{\boldsymbol{r}})$ the three-dimensional over-density of UHECR sources and the UHECR kernel reading

$$
\phi_{\mathrm{CR}}(z):=\left[\int \mathrm{d} \tilde{z} \frac{\alpha(\tilde{z})}{H(\tilde{z})(1+\tilde{z})}\right]^{-1} \frac{\alpha(z)}{(1+z)} .
$$

\subsubsection{Galaxies}

In this case, the key quantity is the projected over-density of sources for a given galaxy sample,

$$
\Delta_{\mathrm{g}}(\hat{\boldsymbol{r}}):=\frac{N_{\mathrm{g}}(\hat{\boldsymbol{r}})-\bar{N}_{\mathrm{g}}}{\bar{N}_{\mathrm{g}}}
$$

where $N_{\mathrm{g}}(\hat{\boldsymbol{r}})$ is the number of galaxies in the direction $\hat{\boldsymbol{r}}$ and $\bar{N}_{\mathrm{g}}$ is its average over the celestial sphere. As is well-known in cosmological studies, Equation 6 is related to the three-dimensional galaxy over-density $\delta_{\mathrm{g}}(z, r \hat{\boldsymbol{r}})$ via the (weighted) distribution of galaxy distances, $\phi_{\mathrm{g}}(r)$, through

$$
\Delta_{\mathrm{g}}(\hat{\boldsymbol{r}})=\int \mathrm{d} r \phi_{\mathrm{g}}(r) \delta_{\mathrm{g}}(z, r \hat{\boldsymbol{r}})
$$

Assuming that redshift information is available for all galaxies in the catalogue-which is the case when dealing with spectroscopically detected galaxies-we can use that information to apply a distance-dependent weight, $w(r)$. In that case, the galaxy over-density kernel $\phi_{\mathrm{g}}(r)$ is given by

$$
\phi_{\mathrm{g}}(r):=\left[\int \mathrm{d} \tilde{r} \tilde{r}^{2} w(\tilde{r}) \bar{n}_{\mathrm{g}, \mathrm{c}}(\tilde{r})\right]^{-1} r^{2} w(r) \bar{n}_{\mathrm{g}, \mathrm{c}}(r)
$$

where $\bar{n}_{\mathrm{g}, \mathrm{c}}$ is the comoving number density of galaxies in the sample. If no redshift information is in fact available, this simply corresponds to applying no weights-namely, setting $w(r)=1$. Then, the normalisation in Equation 8 is just the average surface density of galaxies in the sample, $\bar{N}_{\Omega, \mathrm{g}}$. 


\subsection{Power spectra}

A given observation of the anisotropy fields $\Delta_{a}$, with $a, b \in\{\mathrm{CR}, g\}$, will consist of both signal and noise. If signal and noise are uncorrelated, the resulting observed power spectrum is $C_{\ell}^{a b}:=\mathcal{S}_{\ell}^{a b}+\mathcal{N}_{\ell}^{a b}$, where $\mathcal{S}_{\ell}^{a b}$ and $\mathcal{N}_{\ell}^{a b}$ are the power spectra of signal and noise, respectively. The signals described here are related to the intrinsic clustering of both UHECRs and galaxies, due to the underlying LSS. On the other hand, the noise is sourced by the discrete nature of both tracers.

Now, the harmonic-space power spectrum $\mathcal{S}_{\ell}^{a b}$ between two projected quantities $\Delta_{a}$ and $\Delta_{b}$ is related to their three-dimensional power spectrum $P_{a b}(k, z)$ by

$$
\mathcal{S}_{\ell}^{a b}=\int \mathrm{d} r \frac{\phi_{a}(r) \phi_{b}(r)}{r^{2}} P_{a b}\left[z(r), k=\frac{\ell+1 / 2}{r}\right],
$$

where $\phi_{a}$ and $\phi_{b}$ are the radial kernels of both quantities [see e.g. appendix B of 17]. If the galaxy survey is sufficiently complete, all UHECRs are associated to an observed galaxy, i.e. $\delta_{\mathrm{s}}=\delta_{\mathrm{g}}$.

Finally, both projected overdensities, $\Delta_{\mathrm{CR}}$ and $\Delta_{\mathrm{g}}$, are associated to discrete point processes, represented by the angular positions of the UHECRs and the galaxies in each sample. Therefore, the shot-noise contributions to the power spectra are simply

$$
\mathcal{N}_{\ell}^{\mathrm{CRCR}}=\left(\bar{N}_{\Omega, \mathrm{CR}}\right)^{-1}, \quad \mathcal{N}_{\ell}^{\mathrm{gg}}=\mathcal{N}_{\ell}^{\mathrm{gCR}}=\left(\bar{N}_{\Omega, \mathrm{g}}\right)^{-1} .
$$

Since typically $\bar{N}_{\Omega, \mathrm{CR}} \ll \bar{N}_{\Omega, \mathrm{g}}$, then $\mathcal{N}_{\ell}^{\mathrm{g} C R} \ll \mathcal{N}_{\ell}^{\mathrm{CRCR}}$, and therefore $\mathcal{N}_{\ell}^{\mathrm{gCR}}$ can be neglected. In the case of non-flat galaxy weights, the galaxy-galaxy noise power spectrum reads

$$
\mathcal{N}_{\ell}^{\mathrm{gg}}=\left[\int \mathrm{d} r r^{2} w(r) \bar{n}_{\mathrm{g}, \mathrm{c}}(r)\right]^{-2} \int \mathrm{d} r r^{2} w^{2}(r) \bar{n}_{\mathrm{g}, \mathrm{c}}(r) .
$$

\subsection{Signal-to-noise ratio}

As a figure of merit for the detectability of UHECR anisotropies on a given angular scale, I shall employ the signal-to-noise ratio (SNR) as a function of multipole, defined as

$$
\mathrm{SNR}_{\ell}^{a b}=\sqrt{\frac{N_{(a b)}(2 \ell+1)}{C_{\ell}^{\mathrm{gg}} C_{\ell}^{\mathrm{CRCR}}+\left(C_{\ell}^{\mathrm{gCR}}\right)^{2}}} \mathcal{S}_{\ell}^{\mathrm{gCR}},
$$

where $N_{(a b)}$ represents the number of fields tracing UHECR anisotropies, and is 2 if $a=b=\mathrm{CR}$ and 1 if $a \neq b$. The total SNR for auto- and cross-correlation combined is obtained by considering the cross-covariance between all the terms, as explained in ref. [10]. Note that the cumulative SNR up to a certain $\ell_{\max }$ is the square root of the sum in quadrature of the $\mathrm{SNR}_{\ell}^{a b}$ for each $\ell$ up to $\ell_{\max }$.

\section{Results}

The energy $E_{\text {cut }}$ at which we cut the UHECR integral spectrum determines both the magnitude of the UHECR anisotropic signal and the number of UHECR events. Clearly, there is a trade-off between former and the latter: the lowest $E_{\text {cut }}$, the larger the number of recorded events, because the UHECR spectrum is very steep. On the other hand, at high energies the UHECR horizon is smaller, with UHECRs experiencing smaller deflections due to the intervening magnetic field(s), 
which would otherwise wash out the anisotropy below a characteristic scale. However, such more pronounced anisotropy will be harder to detect due to the much smaller number of events.

Here, I focus on three benchmark scenarios to compare auto- and cross-correlation performance in detecting UHECR anisotropies, based on data currently available [18]: $\left\{E_{\text {cut }} / \mathrm{EeV}, \bar{N}_{\Omega, \mathrm{CR}}\right\}=$ $\left\{10^{19.6}, 1000\right\},\left\{10^{19.8}, 200\right\}$, and $\left\{10^{20}, 30\right\}$. I shall also assume observations over the full sky. This is of course a first approximation, which will nonetheless serve well for our purpose. Besides, I emphasise that most of the crucial pieces of information about UHECR data is well represented in these estimates, i.e. the energy cut and, consequently, the relative UHECR energy losses, expected number of events, and resolution representative of current experiments.

As in ref. [10], I shall here work with a proton-only toy model with $\gamma=2.6$ [see 19, 20]. The attenuation factor has been produced by tracking $10^{6}$ events with energies above $10 \mathrm{EeV}$ (with an upper cutoff at $10^{5} \mathrm{EeV}$ ), for redshifts up to $z=0.3$, and counted the number of events reaching the Earth with $E>E_{\text {cut }}$ for different values of $E_{\text {cut }} \cdot{ }^{1}$ I shall consider a low-redshift galaxy survey modelled after the 2MASS Redshift Survey (2MRS) [23], which constitutes one of the most complete full-sky spectroscopic low-redshift surveys. Then, a halo model prescription for the galaxy Fourier-space power spectrum $P_{\text {gg }}$ will be employed, based on the halo occupation distribution model described in ref. [24] for the 2MRS sample. ${ }^{2}$

\subsection{Power spectra}

Fig. 1 illustrates the expected signal for UHECR auto-correlation ('AC', left panel), its crosscorrelation with 2MRS galaxies ('XC', middle panel) and the same in the case of an optimal redshift weighting scheme (right panel). The three benchmark scenarios introduced above are respectively rendered in red, yellow, and blue colours (see legend), with dashed lines representing the theoretical prediction and solid curves including a 1 deg Gaussian smoothing, to account for the angular resolution of UHECR experiments. On top of it, shaded boxes depict the corresponding $\ell$-binned $1 \sigma$ error bars as for $20 \log$-spaced multipole bins between $\ell_{\min }=2$ and $\ell_{\max }=1000$.

The take-home message here is that the range of multipoles where the error bars are small enough to allow for a detection is wider for the cross-correlation than for auto-correlation for the first two $E_{\text {cut }}$ values. Conversely, for sparser UHECR samples the detectable range of multipoles for the cross-correlation is smaller and pushed towards larger $\ell$ 's compared to the auto-correlation. The reason for this is twofold. First, for the higher UHECR energy end, the propagation horizon of UHECRs is small, and the UHECR sky looks more anisotropic, boosting the auto-correlation. Secondly, the higher the energy, the smaller the overlap of the $\phi_{a}(r)$ kernels in Equation 9, which suppresses the cross-correlation signal—all the more so if we consider that higher energies have also a larger shot noise in the UHECR sample. However, this mismatch between the kernels is removed when optimal weights are applied, with a striking effect on the observations, as can be seen in the rightmost panel of Figure 1.

${ }^{1}$ This has been produced using SimProp [21, v2r4], accounting for energy losses, interactions with cosmic-microwavebackground photons, and with extra-Galactic background photons according to ref. [22].

${ }^{2}$ See [24] and references therein for further details about the specifics of the halo occupation distribution model used. 


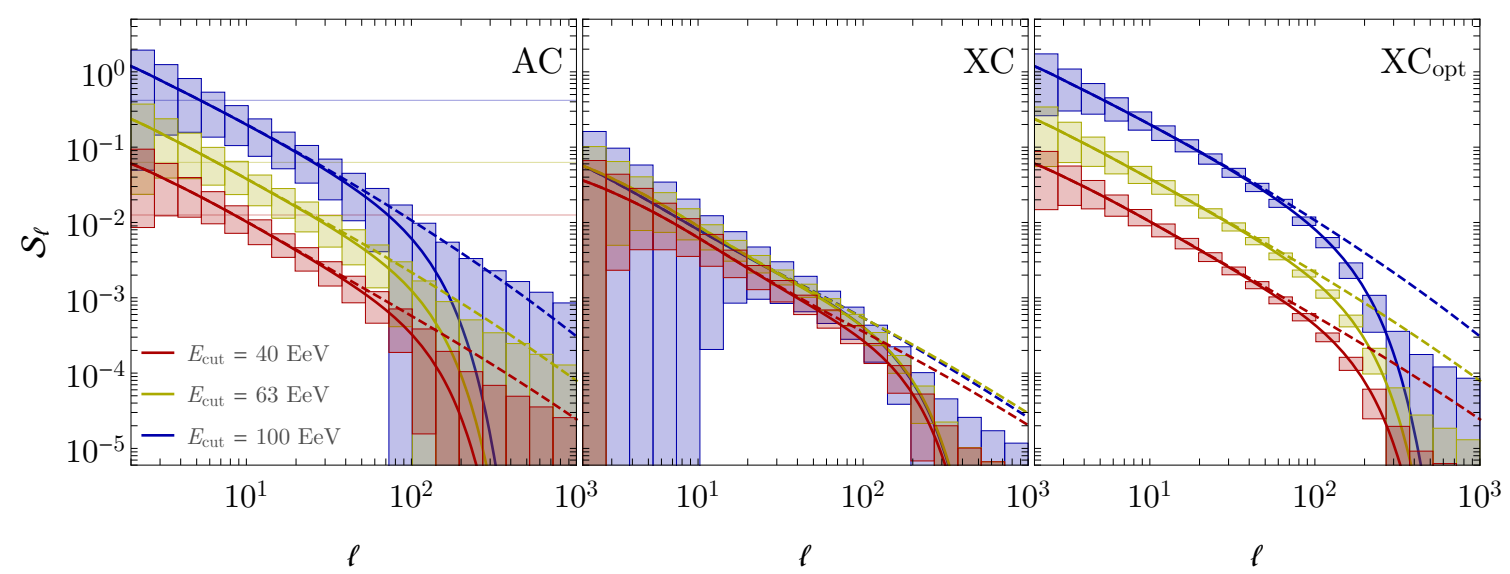

Figure 1: Expected power spectra and $\ell$-binned $1 \sigma$ uncertainties (shaded boxes) including a 1 deg Gaussian smoothing beam to account for the angular resolution of UHECR experiments (solid curves). For reference, horizontal lines in the left panel denote shot-noise levels and dashed curves show the beam-free prediction.

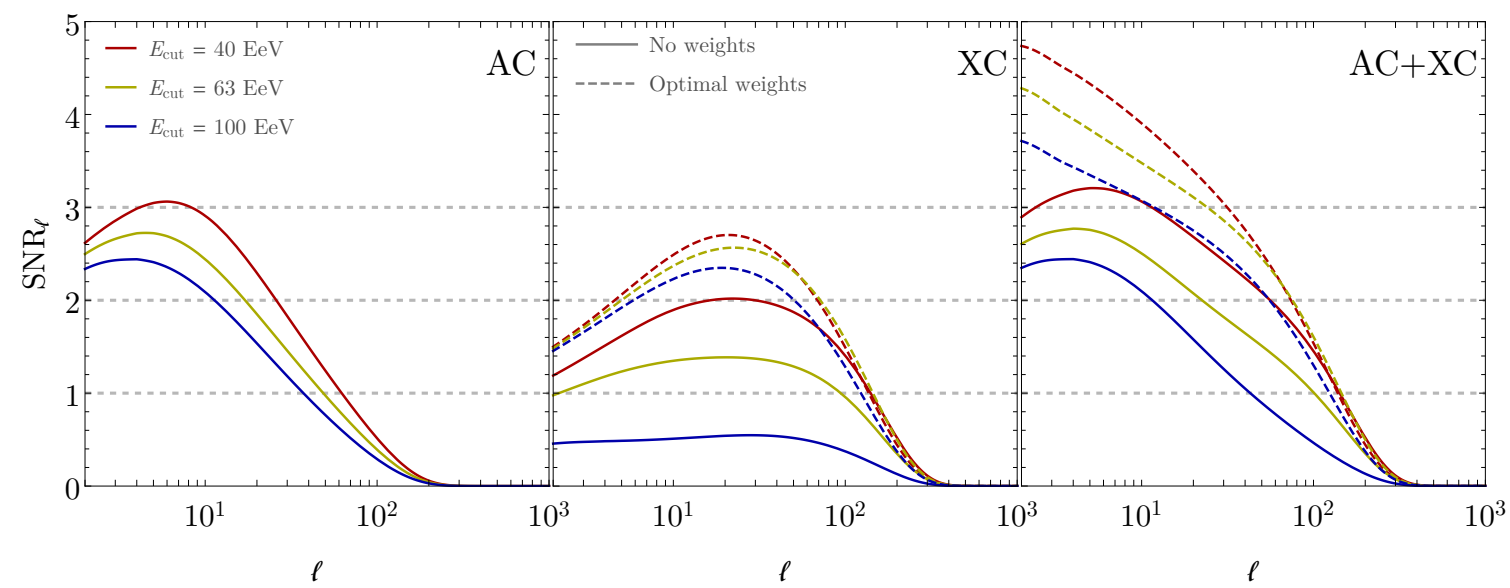

Figure 2: Per-multipole SNR, for the auto- and cross-correlation signals with both normal and optimal weights (left and middle panels), and the combination of the two (rightmost panel). Different colours refer to different energy cuts, and the three horizontal, dashed lines show the thresholds for 1,2 , and $3 \sigma$ detection.

\subsection{Signal-to-noise ratio}

To quantify the improvement in detectability brought by the cross-correlation, in Fig. 2 I show the per-multipole $\mathrm{SNR}, \mathrm{SNR}_{\ell}^{a b}$, with the same colour coding as in Figure 1. Horizontal dashed lines mark the thresholds corresponding to 1,2 and $3 \sigma$ evidence for a one-parameter amplitude fit. These panels can be interpreted as the evidence for anisotropy on a given angular scale, for which it is clear that the cross-correlation with galaxies helps to push the detectability of the signal to smaller scales, under the assumption that UHECRs trace the LSS.

\section{Conclusions}

In this work, I have reviewed a recent piece of work where we introduced a new observable for UHECR physics: the harmonic-space cross-correlation power spectrum between the arrival 
directions of UHECRs and the distribution of the cosmic LSS as mapped by galaxies [10]. Such a new observable can be more easily detected than the UHECR auto-correlation for a range of energies and multipoles (see Figure 1 and Figure 2).

Furthermore, the cross-correlation is more sensitive to small-scale angular anisotropies than the auto-correlation, and viceversa. It can, therefore, be instrumental in understanding properties of UHECR sources that would not be accessible otherwise. Moreover, it is in principle possible to optimise the cross-correlation signal by applying optimal redshift-dependent weights to sources in the galaxy catalogue. This is an aspect that will have to be explored more in detail in the future, as well as a proper modelling of experimental uncertainties and systematic effects, and the impact of magnetic fields. To this end, the cross-correlation, could be employed to understand the properties of the Galactic magnetic field, thanks to its higher SNR and sensitivity to small angular scales.

\section{Acknowledgments}

I warmly thank my co-authors Federico Urban and David Alonso for commencing this interesting journey with me and letting me present results from an ongoing, common project.

\section{References}

[1] J. Linsley, L. Scarsi and B. Rossi, Extremely energetic cosmic-ray event, Phys. Rev. Lett. 6 (1961) 485 .

[2] K. Kotera and A.V. Olinto, The Astrophysics of Ultrahigh Energy Cosmic Rays, Ann. Rev. Astron. Astrophys. 49 (2011) 119 [1101.4256].

[3] Pierre Auger collaboration, Highlights from the Pierre Auger Observatory (ICRC2019), PoS ICRC2019 (2020) 004 [1909. 10791].

[4] P.G. Tinyakov, F.R. Urban, D. Ivanov, G.B. Thomson and A.H. Tirone, A signature of EeV protons of Galactic origin, Mon. Not. Roy. Astron. Soc. 460 (2016) 3479 [1511. 01333].

[5] Pierre Auger collaboration, Observation of a Large-scale Anisotropy in the Arrival Directions of Cosmic Rays above $8 \times 10^{18} \mathrm{eV}$, Science 357 (2017) 1266 [1709.07321].

[6] K. Greisen, End to the cosmic ray spectrum?, Phys. Rev. Lett. 16 (1966) 748.

[7] G.T. Zatsepin and V.A. Kuzmin, Upper limit of the spectrum of cosmic rays, JETP Lett. 4 (1966) 78.

[8] Pierre Auger collaboration, Observation of the suppression of the flux of cosmic rays above $4 \times 10^{19} \mathrm{eV}$, Phys. Rev. Lett. 101 (2008) 061101 [0806.4302].

[9] M. Scrimgeour et al., The WiggleZ Dark Energy Survey: the transition to large-scale cosmic homogeneity, Mon. Not. Roy. Astron. Soc. 425 (2012) 116 [1205. 6812].

[10] F.R. Urban, S. Camera and D. Alonso, Detecting ultra-high energy cosmic ray anisotropies through cross-correlations, Astron. Astrophys. TBD (2021) A?? [2005. 00244]. 
[11] S. Camera, M. Fornasa, N. Fornengo and M. Regis, A Novel Approach in the Weakly Interacting Massive Particle Quest: Cross-correlation of Gamma-Ray Anisotropies and Cosmic Shear, Astrophys. J. 771 (2013) L5 [1212 . 5018].

[12] N. Fornengo and M. Regis, Particle dark matter searches in the anisotropic sky, Front. Physics 2 (2014) 6 [1312 . 4835].

[13] E. Pinetti, S. Camera, N. Fornengo and M. Regis, Synergies across the spectrum for particle dark matter indirect detection: how HI intensity mapping meets gamma rays, 1911.04989.

[14] N. Fornengo, L. Perotto, M. Regis and S. Camera, Evidence of Cross-correlation between the CMB Lensing and the Г-ray sky, Astrophys. J. 802 (2015) L1 [1410. 4997].

[15] E. Branchini, S. Camera, A. Cuoco, N. Fornengo, M. Regis, M. Viel et al., Cross-correlating the $\gamma$-ray sky with Catalogs of Galaxy Clusters, Astrophys. J. Suppl. 228 (2017) 8 [1612.05788].

[16] S. Ammazzalorso et al., Detection of cross-correlation between gravitational lensing and gamma rays, Phys. Rev. Lett. 124 (2020) 101102 [1907. 13484].

[17] D. Alonso, Linear anisotropies in dispersion-measure-based cosmological observables, Phys. Rev. D 103 (2021) 123544 [2103.14016].

[18] R. Alves Batista et al., Open Questions in Cosmic-Ray Research at Ultrahigh Energies, Front. Astron. Space Sci. 6 (2019) 23 [1903.06714].

[19] B.R. d'Orfeuil, D. Allard, C. Lachaud, E. Parizot, C. Blaksley and S. Nagataki, Anisotropy expectations for ultra-high-energy cosmic rays with future high statistics experiments, Astron. Astrophys. 567 (2014) A81 [1401.1119].

[20] A. di Matteo and P. Tinyakov, How isotropic can the UHECR flux be?, Mon. Not. Roy. Astron. Soc. 476 (2018) 715 [1706. 02534].

[21] R. Aloisio, D. Boncioli, A. Di Matteo, A.F. Grillo, S. Petrera and F. Salamida, SimProp v2r4: Monte Carlo simulation code for UHECR propagation, JCAP 1711 (2017) 009 [1705.03729].

[22] F.W. Stecker, M.A. Malkan and S.T. Scully, Intergalactic photon spectra from the far ir to the uv lyman limit for $0<Z<6$ and the optical depth of the universe to high energy gamma-rays, Astrophys. J. 648 (2006) 774 [astro-ph/0510449].

[23] J.P. Huchra, L.M. Macri, K.L. Masters, T.H. Jarrett, P. Berlind, M. Calkins et al., The 2MASS Redshift Survey-Description and Data Release, Astrophys. J. Suppl. 199 (2012) 26 [1108.0669].

[24] S. Ando, A. Benoit-Lévy and E. Komatsu, Angular power spectrum of galaxies in the 2MASS Redshift Survey, Mon. Not. Roy. Astron. Soc. 473 (2018) 4318 [1706.05422]. 